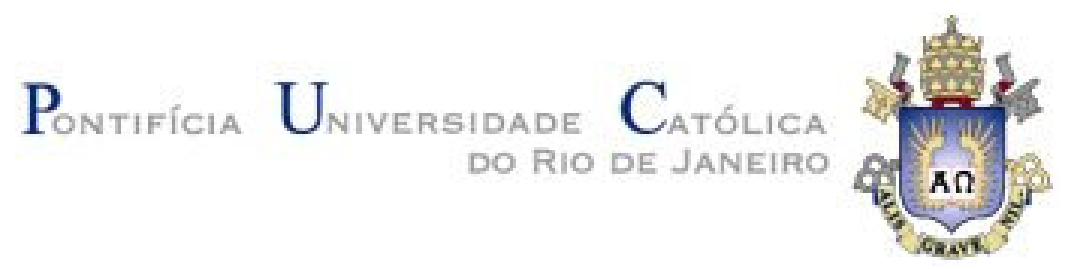

Henrique Prado Sousa

\title{
Integrando modelagem intencional à modelagem de processos
}

\section{Dissertação de Mestrado}

Dissertação apresentada como requisito parcial para obtenção do grau de Mestre pelo Programa de PósGraduação em Informática do Departamento de Informática da PUC-Rio.

Orientador: Prof. Julio Cesar Sampaio do Prado Leite 


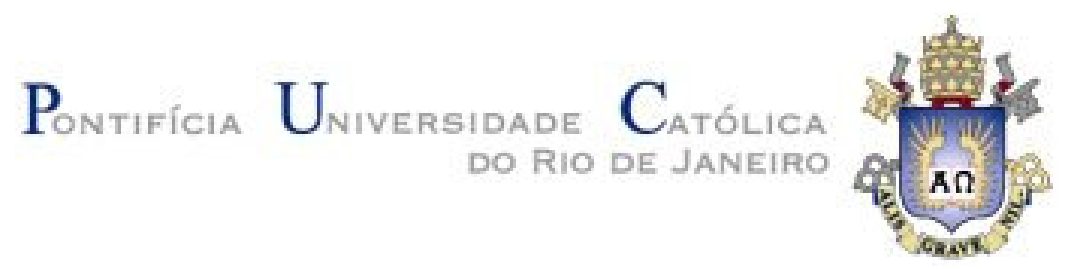

Henrique Prado Sousa

\title{
Integrando modelagem intencional à modelagem de processos
}

\begin{abstract}
Dissertação apresentada como requisito parcial para obtenção do grau de Mestre pelo Programa de PósGraduação em Informática do Departamento de Informática do Centro Técnico Científico da PUC-Rio. Aprovada pela Comissão Examinadora abaixo assinada.
\end{abstract}

Prof. Edward Hermann Haeusler

Presidente

Departamento de Informática - PUC-Rio

Prof. Julio Cesar Sampaio do Prado Leite

Orientador

Departamento de Informática - PUC-Rio

Prof. a Cláudia Cappelli Aló

Departamento de Informática - PUC-Rio

Prof. José Eugenio Leal

Coordenador Setorial do Centro

Técnico Científico - PUC-Rio

Rio de Janeiro, 17 de fevereiro de 2012 
Todos os direitos reservados. É proibida a reprodução total ou parcial do trabalho sem autorização da universidade, do autor e do orientador.

\section{Henrique Prado Sousa}

Graduou-se em Bacharelado em Sistemas de Informação na Universidade Federal do Estado do Rio de Janeiro (UNIRIO) em 2010. Desde 2006 participa do núcleo de pesquisa NP2Tec, da UNIRIO. Desde 2010 participa do grupo de engenharia de requisitos da PUC-Rio, coordenado pelo professor Julio Leite.

Ficha Catalográfica

\section{Sousa, Henrique Prado}

Integrando modelagem intencional à modelagem de processos / Henrique Prado Sousa; orientador: Julio Cesar Sampaio do Prado Leite. - Rio de Janeiro PUC, Departamento de Informática, 2012.

v., $138 \mathrm{f}, ;$ II. ; $30 \mathrm{~cm}$

1. Dissertação (mestrado) - Pontifícia Universidade Católica do Rio de Janeiro, Departamento de Informática.

Inclui referências bibliográficas.

1. Informática - Teses. 2. Modelagem de objetivos 3. Modelagem de processos de negócio. I. Leite, Julio Cesar Sampaio do Prado. II. Pontifícia Universidade Católica do Rio de Janeiro. Departamento de Informática. III. Titulo. 


\section{Agradecimentos}

Não há como iniciar uma página de agradecimento contida em mais um trabalho de grande valor em minha vida, sem colocar Ele em primeiro lugar. Ao meu Deus querido, muito obrigado!

Agradeço aos meus pais todos os esforços empenhados unicamente ao meu crescimento pessoal, muito frequentemente, abdicando a si somente para doar a mim ainda mais carinho e dedicação. Espero estar correspondendo a todas as expectativas, de forma a recompensar justamente tudo o que me foi dado. Eternos agradecimentos!

Em sequencia vem uma pessoa muitíssimo especial para mim. Aquela que iniciou, propiciou e alavancou juntinho de mim toda essa vida de nerd que só iniciou depois de quase 20 anos de pura diversão. À minha noiva linda, agradeço de coração!

Agradeço aquelas pessoas que me motivam todos os dias em minha vida, e mal sabem que são meus verdadeiros heróis que eu quero ser quando crescer! Aos meus eternos orientadores Julio Leite, Flávia Santoro e Leonardo Azevedo, Muito obrigado!

Agradeço aos amigos por sempre estarem ao meu lado, motivando, agregando e dedicando à construção deste trabalho! Aos amigos André, Eduardo, Herbet, Leandro, Marx, e todo o grupo de Engenharia de Requisitos da PUC-Rio, broadcast de agradecimentos!!!

E finalmente, agradeço à PUC-Rio, pelos auxílios concedidos, sem os quais este trabalho não poderia ter sido realizado. 


\section{Resumo}

Sousa, Henrique Prado; Leite, Julio Cesar Sampaio do Prado. Integrando modelagem intencional à modelagem de processos. Rio de Janeiro, 2012. 138p. Dissertação de Mestrado - Departamento de Informática, Pontifícia Universidade Católica do Rio de Janeiro.

A modelagem de processos de negócio é utilizada por empresas que desejam documentar detalhes do fluxo de execução de seus processos, resultando em um documento rico em detalhes sobre o negócio. Este artefato também é utilizado pela Engenharia de Software para elicitação de requisitos de sistema. A modelagem intencional possui foco na modelagem de objetivos - definidos como metas e metas flexíveis - e registra as estratégias que podem ser seguidas por um ator de forma a melhor atender suas necessidades, mapeando tarefas e recursos necessários, além disso, também aborda as dependências entre atores. É importante que os modelos de processos de negócio estejam alinhados aos objetivos da organização de forma a prover fonte de informações confiável que gere consequentemente requisitos alinhados ao negócio. Diversas ferramentas estão disponíveis no mercado com o objetivo de apoiar a modelagem dos processos de negócio e dos objetivos organizacionais, entretanto, percebe-se que as soluções disponíveis ainda são incompletas quando se fala na integração de modelos de processos e modelo de objetivos e formas de verificação do alinhamento entre processos e objetivos organizacionais a partir da modelagem. $\mathrm{Na}$ arquitetura organizacional, processos de negócio e objetivos são intrinsecamente interdependentes, porém, as linguagens de modelagem atuais não oferecem recursos suficientes para tratar processos e objetivos de forma alinhada, uma vez que existem deficiências na integração entre a camada de modelagem de objetivos e a de processos. Assim, o uso do ferramental disponível que se apoia nessas linguagens e métodos dificulta sobremaneira a tarefa de identificar se os processos utilizados para gerar serviços e produtos, verdadeiramente atingem os objetivos da organização, bem como o impacto que as mudanças nos objetivos 
causariam nos processos de negócio. Neste trabalho integramos uma linguagem de modelagem de objetivos a uma linguagem de processos de negócio e provemos os elementos e métodos necessários para ampliar a capacidade de análise do alinhamento dos processos de negócio às estratégias organizacionais.

\section{Palavras-chave}

Engenharia de requisitos; modelagem de processos de negócio; modelagem de objetivos; BPM; BPMN; i*; integração de processos e objetivos; alinhamento de processos e objetivos; KPI; indicadores. 


\section{Abstract}

Sousa, Henrique Prado; Leite, Julio Cesar Sampaio do Prado (Advisor). Integrating intentional modeling to process modeling. Rio de Janeiro, 2012. 138p. MSc. Dissertation - Departamento de Informática, Pontifícia Universidade Católica do Rio de Janeiro.

The business processes modeling is used by companies who wish to document details of the execution flow of their processes, resulting in a document rich in details about the business. This artifact is also used by the Software Engineering for system requirements elicitation. The intentional modeling is focused on objectives - defined as goals and softgoals - and registers the strategies that may be followed by an actor in a way to better meet their needs, mapping the tasks and resources needs, in addition, it also addresses the dependencies between actors. It is important that business processes models are aligned to the objectives of the organization in order to provide reliable information source that generates consequently requirements aligned to business. Several tools are available on the market in order to support the business processes and organizational objectives modeling, however, it's possible to realize that the available solutions are still incomplete when it comes to the integration of process models and goals models and ways to check the alignment between organizational goals and processes using the models. In the organizational architecture, business processes and goals are intrinsically interdependent, however, the current modeling languages treat process and goals in a misaligned way, since there are deficiencies in the integration between the modeling layer of objectives and processes. Thus, the use of the available tools that supports these language and methods greatly complicates the task of identify if the processes used to generate products and services truly achieve the organizational goals as well as the impact of the changes in the goals would cause in business processes. In this paper we integrated a goal modeling language to a business processes modeling language and proved the elements and methods needed to expand the capacity of analysis of the alignment 
between the business processes and the organizational strategies.

\section{Keywords}

Requirements engineering; business processes modeling; objectives modeling; BPM; BPMN; $\mathrm{i}^{*}$; integration of processes and goals; alignment of processes and goals; KPI; indicators. 


\section{Sumário}

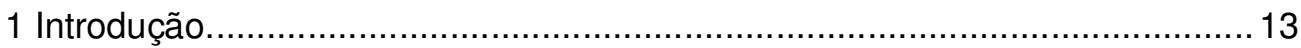

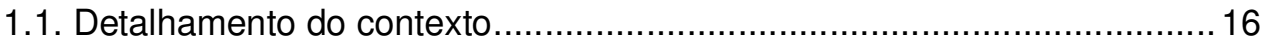

1.2. Abordagem proposta ................................................................ 18

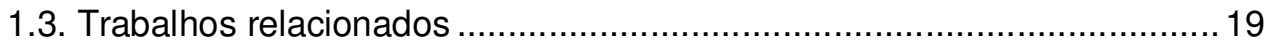

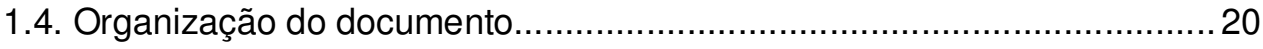

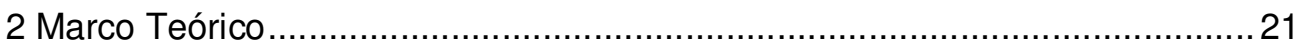

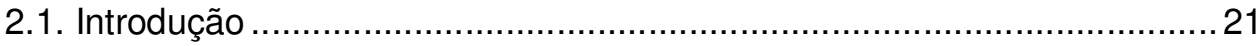

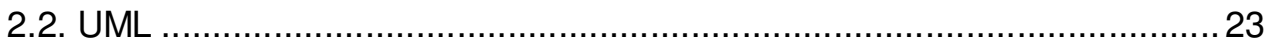

2.2.1. Extensões do diagrama de processo ...........................................24

2.2.2. Extensões do diagrama de objetivos............................................ 28

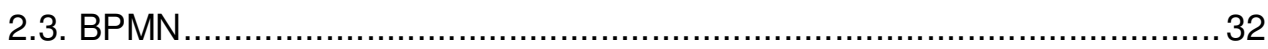

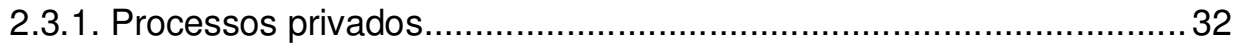

2.3.2. Processos públicos ..................................................................... 33

2.3.3. Processos colaborativos ................................................................. 33

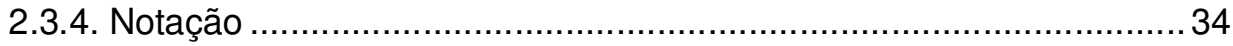

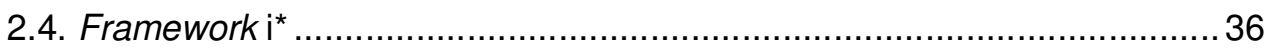

2.4.1. Strategic Dependency (Modelo SD) .............................................. 37

2.4.2. Strategic Rationale (Modelo SR) ............................................. 40

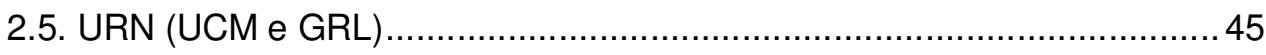

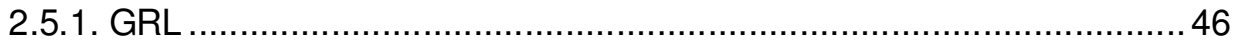

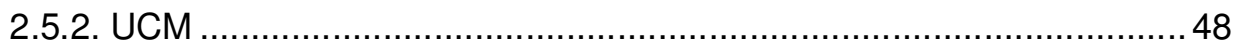

2.5.3. Rastreabilidade entre UCM e GRL............................................ 51

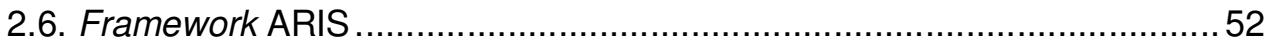

2.6.1. VAC - Valued Added Chain .......................................................... 53

2.6.2. EPC - Event-driven Process Chain .............................................. 54

2.6.3. FAD - Function Allocation Diagram .......................................... 55

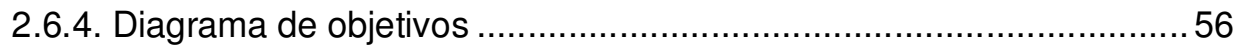




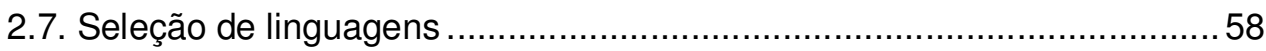

2.7.1. Domínio de processos de negócio .............................................59

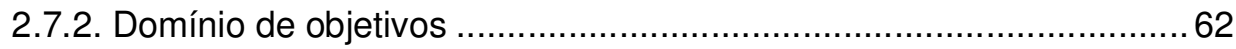

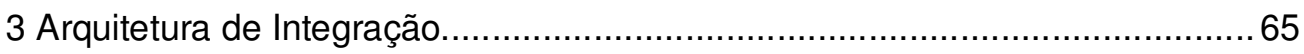

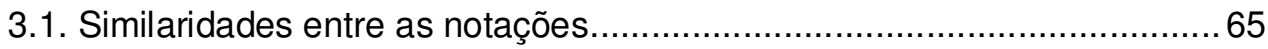

3.2. Adaptações necessárias ......................................................... 68

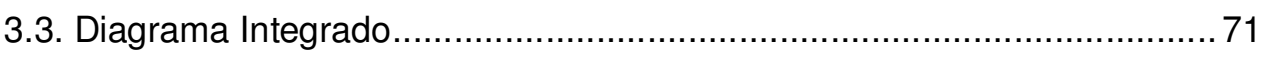

3.4. Análise do alinhamento através de KPIs ............................................. 75

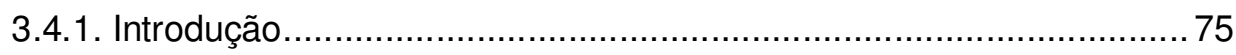

3.4.2. Uso de indicadores no Diagrama Integrado ................................. 78

3.4.3. Uso do Diagrama de Indicadores............................................. 81

4 Implementação da Integração........................................................... 85

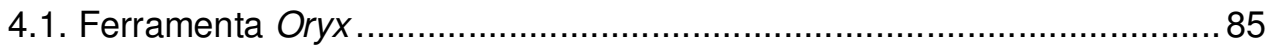

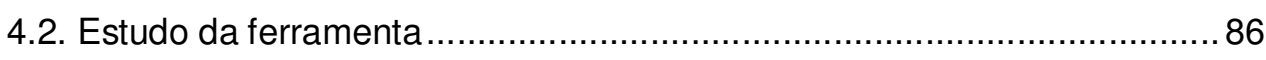

4.2.1. Aplicando a Engenharia Reversa ................................................ 88

4.2.2. Reengenharia de software .................................................. 100

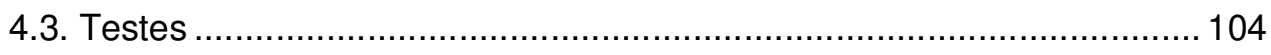

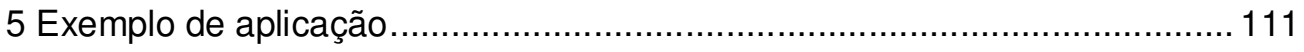

5.1. Modelo de processo ............................................................. 111

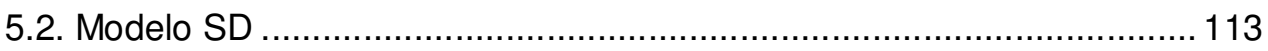

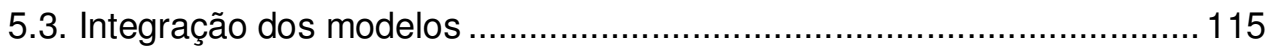

5.4. Modelagem dos indicadores...................................................... 117

5.5. Análise e desenvolvimento de relatórios............................................. 119

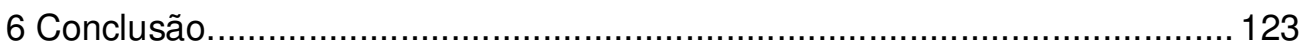

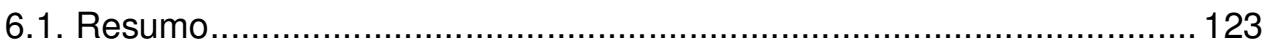

6.2. Comparação com trabalhos relacionados........................................... 125

6.3. Contribuições para Transparência do Processo ................................. 126

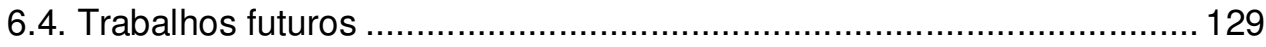

7 Referências bibliográficas ............................................................. 130 


\section{Lista de figuras}

Tabela 1 - Elementos de um diagrama de processo de negócio em UML...................25

Tabela 2 - Elementos de um diagrama de objetivos em UML ............................... 28

Tabela 3 - Recursos e regras compõem todos os diagramas.................................. 31

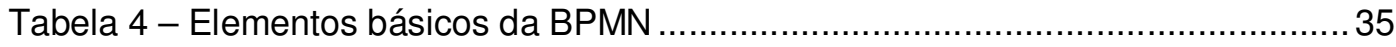

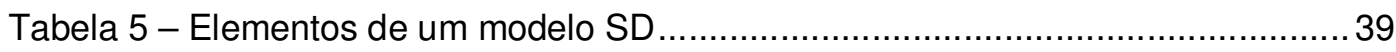

Tabela 6 - Elementos do modelo SR .......................................................... 43

Tabela 7 - Elementos do modelo GRL ........................................................ 47

Tabela 8 - Elementos do modelo UCM........................................................... 49

Tabela 9 - Elementos de um diagrama de objetivos ..............................................56

Tabela 10 - Conexões permitidas entre os elementos do diagrama de objetivos .......57

Tabela 11 - Comparação entre as linguagens de modelagem de processos...............64

Tabela 12 - Comparação entre as linguagens de modelagem de objetivos................. 64

Tabela 13 - Similaridades entre elementos do $i^{\star}$ e BPMN ...........................................6 66

Tabela 14 - Novos elementos incluídos ao Diagrama Integrado...............................78

Tabela 15 - Elementos do Diagrama de Indicadores ............................................ 82

Tabela 16 - Projeção de macroatividades da engenharia reversa da

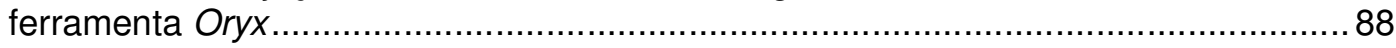

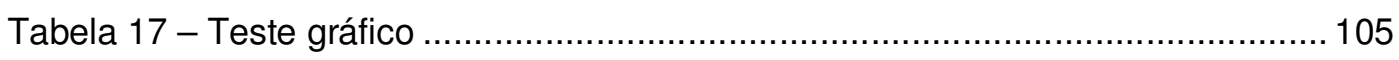

Tabela 18 - Teste de regras do tipo Containment Rules....................................... 106

Tabela 19 - Teste de regras do tipo Cardinality Rules............................................ 106

Tabela 20 - Teste de regras do tipo Connection Rules ......................................... 107

Tabela 21 - Teste gráfico para o elemento Agent (Diagrama SD) ........................... 107

Tabela 22 - Teste gráfico para o elemento meta (Diagrama SD) ........................... 108

Tabela 23 - Teste da regra Containment Rule para o elemento

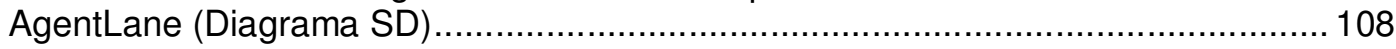

Tabela 24 - Teste da regra Cardinality Rule para os elementos do tipo Evento inicial.

Tabela 25 - Teste da regra Connection Rule para os elementos que utilizam o relacionamento Dependency (Diagrama SD) ..................................................... 110

Tabela 26 - Detalhamento dos indicadores inseridos no Diagrama Integrado ........... 117

Tabela 27 - Resumo de associação entre principais elementos ............................. 120

Tabela 28 - Tabela de descrição de indicadores .................................................. 121

Tabela 29 - Tabela de descrição do recurso crítico ............................................... 121

Tabela 30 - Detalhamento de recursos críticos não identificados no processo.......... 122

Tabela 31 - Correlação entre recursos críticos e papéis responsáveis..................... 122 


\section{Lista de tabelas}

Tabela 1 - Elementos de um diagrama de processo de negócio em UML....................25

Tabela 2 - Elementos de um diagrama de objetivos em UML ................................28

Tabela 3 - Recursos e regras compõem todos os diagramas.................................. 31

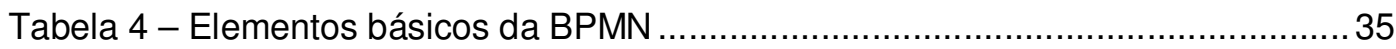

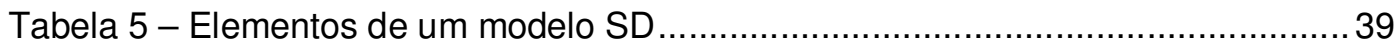

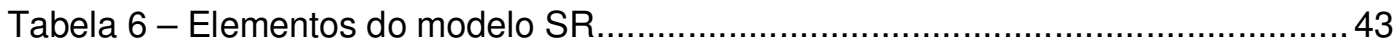

Tabela 7 - Elementos do modelo GRL ..................................................... 47

Tabela 8 - Elementos do modelo UCM............................................................ 49

Tabela 9 - Elementos de um diagrama de objetivos ............................................ 56

Tabela 10 - Conexões permitidas entre os elementos do diagrama de objetivos .......57

Tabela 11 - Comparação entre as linguagens de modelagem de processos.............. 64

Tabela 12 - Comparação entre as linguagens de modelagem de objetivos.................64 64

Tabela 13 - Similaridades entre elementos do $i^{\star}$ e BPMN ......................................66

Tabela 14 - Novos elementos incluídos ao Diagrama Integrado.................................. 78

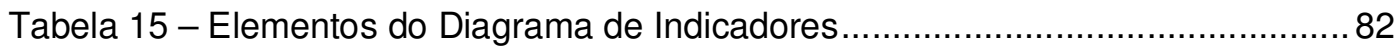

Tabela 16 - Projeção de macroatividades da engenharia reversa da

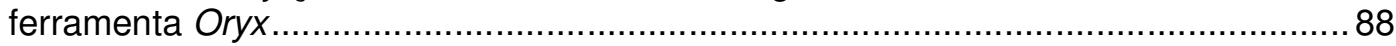

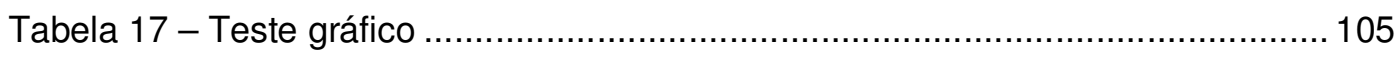

Tabela 18 - Teste de regras do tipo Containment Rules...................................... 106

Tabela 19 - Teste de regras do tipo Cardinality Rules.......................................... 106

Tabela 20 - Teste de regras do tipo Connection Rules ........................................... 107

Tabela 21 - Teste gráfico para o elemento Agent (Diagrama SD) …......................... 107

Tabela 22 - Teste gráfico para o elemento meta (Diagrama SD).............................. 108

Tabela 23 - Teste da regra Containment Rule para o elemento

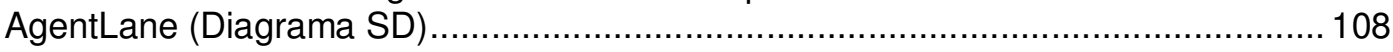

Tabela 24 - Teste da regra Cardinality Rule para os elementos do tipo Evento inicial...

Tabela 25 - Teste da regra Connection Rule para os elementos que utilizam o relacionamento Dependency (Diagrama SD) ................................................... 110

Tabela 26 - Detalhamento dos indicadores inseridos no Diagrama Integrado .......... 117

Tabela 27 - Resumo de associação entre principais elementos ............................. 120

Tabela 28 - Tabela de descrição de indicadores ................................................ 121

Tabela 29 - Tabela de descrição do recurso crítico ................................................ 121

Tabela 30 - Detalhamento de recursos críticos não identificados no processo.......... 122

Tabela 31 - Correlação entre recursos críticos e papéis responsáveis..................... 122 